\title{
Retrospective Identification of Bull and Bear Markets: A new, but simple Algorithm
}

\author{
Marcel Hoepfner
}




\title{
Retrospective Identification of Bull and Bear Markets: A new, but simple Algorithm
}

\author{
Marcel Hoepfner*
}

\begin{abstract}
There are different approaches for the detection of market phase changes in stock markets. Most of them utilize various assumptions and constraints which makes these methods somewhat arbitrary. This paper develops an algorithm which identifies bull and bear markets retrospectively in a very robust way without using exogenous parameters. At the same time the algorithm is very easy to execute, can be applied to several time series frequencies and is intended to identify rather longer subperiods than shorter ones. Knowing the different phases one can investigate a multitude of characteristics of bull and bear markets.
\end{abstract}

Keywords: bull market, bear market, time series, algorithm, market phase changes

JEL classification: C14, C58, G10

Department of Business Administration and Economics, Bielefeld University, Germany.

E-mail: mhoepfner@wiwi.uni-bielefeld.de 


\section{Introduction}

For financial time series a prominent pattern is to find out whether different assets do or do not correlate (positively or negatively) with each other. For this analysis of time series, or rather their characteristics, it is of great interest whether such relations exist in general or only during particular periods of time. Thus, it is important to divide an entire time series in subperiods, which represents particular market phases. These subperiods (or market phases) can be in the form of bull and bear markets, which are here solely defined as increasing and decreasing stock prices. So, the topic of this paper is the development of an algorithm for the identification of bull and bear markets, which therefore is simply called the IBB algorithm. In the present paper it is only applied to time series of different stock market indices, but it is also applicable to time series of individual stocks. Besides, the IBB algorithm can be applied to various data frequencies, which is shown below by analyzing daily, weekly and monthly closing prices. At the same time the resulting market phases are very robust.

There exist different approaches for the identification of bull and bear markets. The one which inspired the IBB algorithm most comes from Pagan and Sossounov (2003), whose basic idea consists of the detection of local maxima and minima as turning points between bull and bear markets. Although this basic idea is often used by further authors (see e.g. Candelon et al., 2008; Coudert and Raymond-Feingold, 2011; Gonzalez et al., 2006; Wu and Lee, 2012) and is also used in the IBB algorithm, the IBB algorithm constitutes a new approach as we try to simplify the existing approaches. Commonly, existing approaches postulate that a market phase - so a bull or a bear market - has to last for at least a certain amount of time so that it can be identified as a separate market phase and can be called a bull or a bear market. This certain amount of time is exogenous in the existing approaches and is endogenous in the IBB algorithm like is shown below. This considerably reduces the arbitrariness of the results as there is no consensus in the literature of how long a market phase has to last so that it can be called a separate market phase.

The algorithm of Pagan and Sossounov (2003) requires that a market phase has to last for at least four months. Otherwise it is not yet a separate market phase, but only a market adjustment during a longer lasting market phase. Furthermore, a cycle - consisting of one bull and one bear market - has to last for at least 16 months. Why they choose just these values for a market phase and a cycle is somewhat nebulous. Though they say that the minimum duration of a market phase 
is based on the well known work of Hamilton (1919) and the deduced minimum duration of three months, they do not explain why they choose the deviating four months or why they think that the three months of Hamilton (1919) would or would not be adequate as minimum duration. A similar criticism applies to cycles lasting 16 months. As Pagan and Sossounov (2003) describe that the duration of a cycle is leaned on the 15 months duration of business cycles, they do not say why a cycle of bulls and bears can be compared with business cycles. This question arises especially because expansions and contractions do not conform with bull and bear markets (see e.g. Chauvet and Potter, 2000; Gonzalez et al., 2006). A further look in the relevant literature provides evidence of the arbitrariness of the choice of the minimum duration. Although Pagan and Sossounov (2003) are inspired by Bry and Boschan (1971), Bry and Boschan (1971) use in their algorithm a minimum duration of five months for a market phase and 15 months for a cycle. However, their algorithm was created for the detection of business cycles and so for the identification of expansions and contractions. Candelon et al. (2008) postulate six months as the minimum duration for a market phase, whereas Hardouvelis and Theodossiou (2002) implement the option to choose due to the readers preferences between three, four, five or six months as minimum duration for a market phase. As a matter of fact, it could be useful to allow the user of an algorithm to choose if rather short-term or rather long-term market phases are to be identified. Thus the user could adjust the algorithm so that it meets his need for the identification of shorter or longer subperiods - depending on whether he is more interested in shorter or longer effects.

Other papers postulate a broad increase or decrease of stock prices so that a separate market phase can be identified. Pagan and Sossounov (2003) e.g. allow - in spite of the postulated minimum duration - to identify a market phase which is shorter than four months if stock prices increase or decrease more than $20 \%$ in under four months. But the choice of $20 \%$ as the minimum increase or decrease is as arbitrary as the choice of four months as the minimum duration. Pagan and Sossounov (2003) themselves say that bull (bear) markets are characterized by an increase (decrease) of $20 \%$ to $25 \%$, so that the choice of just $20 \%$ - and therefore not $25 \%$ e.g. is questionable. Other authors prefer differing values as the minimum price variation. Lunde and Timmermann (2004), for example, use a decrease of $10 \%$ to $15 \%$ for the identification of bear markets and an increase of $15 \%$ to $20 \%$ for the identification of bull markets. Gonzalez et al. (2006), in contrast, are content with a price variation of just $10 \%$ to identify a separate market phase. In none of the cases it can be said why exactly these certain values are chosen. To avoid 
such kind of arbitrariness the IBB algorithm does not postulate a certain increase or decrease of stock prices.

The IBB algorithm is of a much simpler construction because less constraints have to be considered. Hence, it is much easier to implement and to work with - and still significantly less arbitrary. Furthermore, it is to mention that even authors who postulate minimum durations or minimum price variations are to some extent aware of the arbitrariness of their approaches.

The rest of this article is organized as follows. Section 2 presents the method of the IBB algorithm including a general definition of bull and bear markets. In section 3 the algorithm is applied to various financial time series to illustrate the applicability as well as the robustness of the algorithm and its results, respectively. And finally, section 4 concludes the paper.

\section{Method}

As already mentioned the basic idea is to detect local maxima and minima. Local maxima represent a turn from a bull to a bear market and local minima represent a turn from a bear to a bull market. So, local maxima are the beginning of a bear market and local minima are the beginning of a bull market. This is due to the widely referred link between bull (bear) markets and increasing (decreasing) stock prices (see e.g. Candelon et al., 2008; Coudert and Raymond-Feingold, 2011; Pagan and Sossounov, 2003). Authors who postulate a mimimum duration or minimum price variation use in their definition of bull (bear) markets also increasing (decreasing) stock prices. While these links are now commonly accepted, the above mentioned minimum durations and minimum price variations are not. Hence, bull (bear) markets are here solely defined by generally increasing (decreasing) prices. Both kinds of market phases are comprehended as longer lasting periods of time so that each market phase can - and will - contain countermovements without already initializing a new market phase. This is very important because of the typical frequently occurring ups and downs in financial time series - especially looking at daily prices.

Each identified beginning of a market phase represents the end of the contrary market phase. Because it cannot be said if the corresponding data point is the beginning or the end of a market phase, each local extremum is at the same time both a beginning and an end of a market phase. Thus the corresponding data point is part of two market phases. Now, to detect the local extrema for the identification of longer lasting market phases a window is set symmetrically around each 
data point $i$ with $i \in \mathbb{N}$ and for each such window both the local maximum and the local minimum is determined. The data points can be of different frequencies like daily, weekly and monthly data for example. The window size is variable due to the purpose of investigation. If one wants to identify rather longer subperiods a larger window size should be chosen, whereas one should choose smaller window sizes if one is more interested in shorter subperiods. By choosing larger window sizes less local extrema and hence less changes between bull and bear markets can be detected. In the present paper longer subperiods are requested and therefore larger window sizes are chosen. A great benefit of larger window sizes is a higher robustness of the results against changes of the exact window size. To minimize the arbitrariness of choosing the window size, the window size with the highest possible robustness against changes of this size has to be taken. So, there is no (arbitrary) choice of the window size, but the window size to be used is endogenous determined by the most robust one.

The total window size - measured in trading days or data points, respectively - is called $F$ and has to be an odd number of data points due to its symmetry. $f$ with $f:=\frac{F-1}{2}$ denominates the number of data points which are considered previously and afterwards the data point, which represents the symmetrical center around which the window is set. Only deviations from this shall be made, if one sets a window around a data point $i$, which is too close to the start (end) of the time series so that there are not $f$ data points previously (afterwards) this data point. In this case as many data points as existent are considered previously (afterwards) of data point $i$. To ensure that $F$ is an odd number, the most robust $f$ is determined and following from this one calculates $F$ with $F=f \cdot 2+1$. The determination of the exact value of the most robust $f$ is the last step of the IBB algorithm and thus, this step will follow later on.

In the first step of the IBB algorithm for each such window the local maxima $(\operatorname{Max}(i))$ and local minima $(\operatorname{Min}(i))$ are detected. $N$ denominates the total number of observations $i$ or rather stock prices $S_{i}$ :

$$
\begin{aligned}
\operatorname{Max}(i) & :=\max _{j \in\{\max \{1 ; i-f\}, \ldots, \min \{i+f ; N\}\}} S_{j} \quad, \quad 1 \leq i \leq N \\
\operatorname{Min}(i) & :=\min _{j \in\{\max \{1 ; i-f\}, \ldots, \min \{i+f ; N\}\}} S_{j} \quad, \quad 1 \leq i \leq N
\end{aligned}
$$

Figure 1 shows an exemplary illustration of the identification of local maxima with the help of the described windows. 


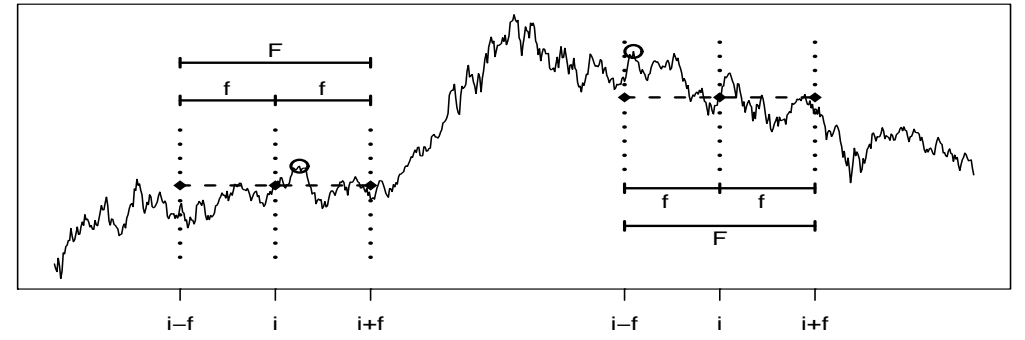

Figure 1. Illustration of the usage of windows to identify maxima. The figure shows exemplarily for two data points $i$ the idea of the identification of local maxima with the help of the described windows. The windows are set symmetrically around each data point $i$. Based on the data point $i$, all $f$ data points before and after this data point $i$ are considered by the window of total size $F$. Thus, the start and the end of the window which is set symmetrically around data point $i$ is located at data point $i-f$ and $i+f$, respectively. The vertical dotted lines describe the data points $i-f, i$ and $i+f$, the horizontal dashed lines describe the total window size and the circles describe the maxima of the corresponding windows.

In the next step of the IBB algorithm the function $L_{i}^{\text {Max }}\left(L_{i}^{\text {Min }}\right)$ in equation (3) ((4)) counts the number of consecutive windows, for which the same local maxima (minima) are identified. This counting algorithm is starting from 1 and adds 1 for each following consecutive window with the same maximal (minimal) value. If two consecutive windows exhibit different maximal (minimal) values, the counting algorithm starts from 1 again, and so forth. Once the counting algorithm reaches $F$ - meaning that $F$ consecutive windows exhibit the same maxima (minima) the data point which is located $f$ data points before the data point for which the counting algorithm reaches $F$ represents the identified maximum (minimum). This is because this data point is just the symmetrical center of the window for which all $f$ preceding and all $f$ following data points exhibit one and the same maximal (minimal) value. So, the counting algorithm identifies in equation (5) ((6)) a maximum (minimum) at data point $i$, if the function $L_{i}^{\operatorname{Max}}\left(L_{i}^{\operatorname{Min}}\right)$ reaches $F$ at data point $i+f$.

In opposition to the fact that local maxima and minima have to alternate, here financial time series can feature successive local maxima without a local minimum in between and likewise they can feature successive local minima without a local maximum in between. But a bull market has necessarily to go from a local minimum up to a local maximum and a bear market has necessarily to go from a local maximum down to a local minimum. Therefore, multiple successive local maxima (minima) has inevitably to be eliminated so that local maxima and minima are alternating again. Every local maximum (minimum) which is identified by $L_{i}^{\operatorname{Max}}\left(L_{i}^{\operatorname{Min}}\right)$ is hence merely a possible beginning of a bear (bull) market. Formally, a possible beginning of a bear market at data 
point $i\left(\right.$ BeginBear $\left._{i}^{\text {pos }}\right)$ and a possible beginning of a bull market at data point $i\left(\right.$ BeginBull $\left._{i}^{\text {pos }}\right)$ are determined as follows:

$$
\begin{aligned}
& L_{i}^{\operatorname{Max}}:=\left\{\begin{array}{lll}
1 & \text { if } \quad i=1 \\
1 & \text { if } \quad \operatorname{Max}(i) \neq \operatorname{Max}(i-1), 2 \leq i \leq N \\
L_{i-1}^{\operatorname{Max}}+1 & \text { if } \quad \operatorname{Max}(i)=\operatorname{Max}(i-1), 2 \leq i \leq N
\end{array}\right. \\
& L_{i}^{M i n} \quad:=\left\{\begin{array}{lll}
1 & \text { if } \quad i=1 \\
1 & \text { if } \quad \operatorname{Min}(i) \neq \operatorname{Min}(i-1), 2 \leq i \leq N \\
L_{i-1}^{M i n}+1 & \text { if } \quad \operatorname{Min}(i)=\operatorname{Min}(i-1), 2 \leq i \leq N
\end{array}\right. \\
& \text { BeginBear }_{i}^{\text {pos }}:= \begin{cases}1 & \text { if } L_{i+f}^{M a x}=F, 1 \leq i \leq N \\
0 & \text { else }, 1 \leq i \leq N\end{cases} \\
& \text { BeginBull }_{i}^{\text {pos }}:= \begin{cases}1 & \text { if } L_{i+f}^{\text {Min }}=F, 1 \leq i \leq N \\
0 & \text { else }, 1 \leq i \leq N\end{cases}
\end{aligned}
$$

Figure 2 gives an exemplary illustration of the counting algorithm for the identification of possible beginnings of a bear market.

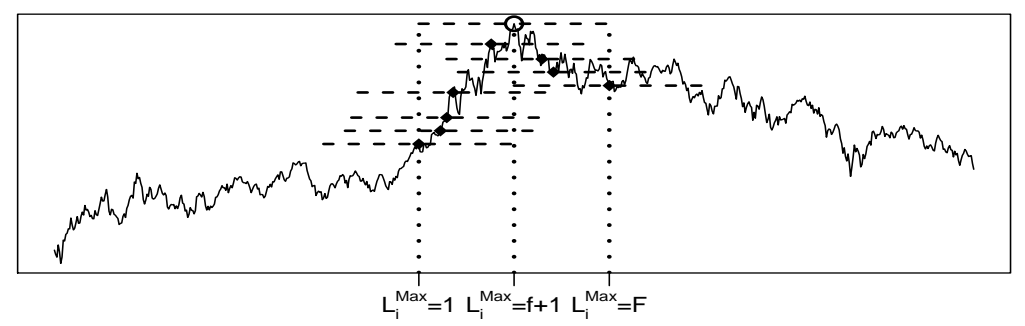

Figure 2. Illustration of the counting algorithm for the identification of possible beginnings of bear markets. The eight dashed horizontal lines illustrate eight representatives of the windows which are set symmetrically around each data point $i$. All these representatives identify one and the same possible local maximum which is described by the circle and the second dotted vertical line. So it represents a possible beginning of a bear market, which is identified by the counting algorithm. The two other dotted vertical lines describe the beginning and the end of the window which is set around this data point. The data point marked by the circle is identified as a possible beginning of a bear market because all windows which are set symmetrically around all $f$ data points before and after this data point identify the data point marked by the circle as the maximal value. Thus, $f$ data points before it the counting algorithm $L_{i}^{M a x}$ starts at 1 . Because all $F$ succeeding windows identify the same maximal value, the counting algorithm reaches $f+1$ at the possible local maximum and $F$ at $f$ data points after it. This is equivalent to the identification of a possible beginning of a bear market at data point $i$ if the counting algorithm reaches $F$ at data point $i+f$. 
In the case of already alternating local maxima and minima, a local maximum (minimum) lies automatically inside of two local minima (maxima). All possible beginnings of bull (bear) markets are then immediately actual ones and no elimination of successive local minima (maxima) is necessary. Exceptional cases are the first and last identifiable local extrema of the entire time series, which cannot have a preceding or a following extremum, respectively. If in contrast successive possible local maxima (minima) exist, then - following the idea of Pagan and Sossounov (2003) and Bry and Boschan (1971) - the highest (lowest) of the successive possible local maxima (minima) constitute the actual local maximum (minimum). All hereby eliminated possible local extrema are just countermovements or market adjustments inside a longer lasting market phase, but not the beginning of a separate market phase itself. It should be noted again that the beginning of a market phase implies that until this data point the contrary market phase persists. Figure 3 depicts an exemplary illustration of the application of the described elimination algorithm.

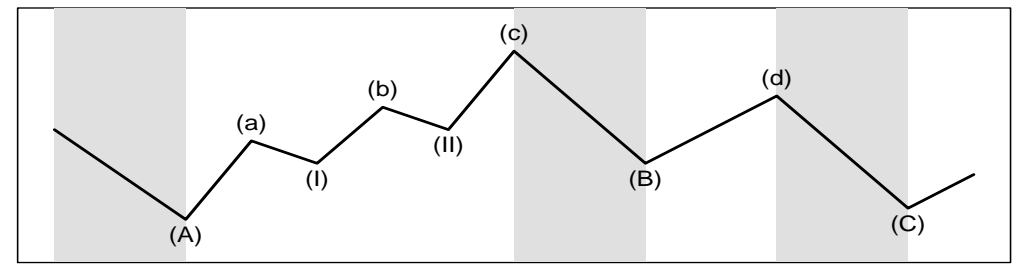

Figure 3. Illustration of the elimination algorithm for successive possible beginnings of bear markets. Suppose that the IBB algorithm identifies in its first step the data points (a), (b), (c) and (d) as possible beginnings of bear markets and the data points (A), (B) and (C) as possible beginnings of bull markets, whereas the data points (I) and (II) were not identified as possible beginnings of a bull market and, therefore, represent only countermovements. Thus (a), (b) and (c) are obviously successive possible beginnings of bear markets, so that the actual one has to be determined. The elimination algorithm would identify (c) as the actual beginning of a bear market, because it is the highest of the successive possible beginnings of bear markets. Bear markets are represented by shaded areas and bull markets by non shaded areas.

Now, the most robust $f$ can be determined. To do so the IBB algorithm has to be executed for a multitude of values for $f$. The more consecutive values for $f$ result in the same bull and bear markets identified by the IBB algorithm, the more robust are the final results. This means that one has to ascertain the range of $f$ for which the same results occur and that the largest range of $f$ with the same occurring results is the most robust one. If this most robust range considers an odd number of observations then the median of this range - which is equivalent to the arithmetic mean of the limits of this range - represents the most robust $f$. In the case of an even number of observations the rounded down arithmetic mean of the limits of the most robust range is the most 
robust $f$. Using finally the most robust $f$ for the IBB algorithm leads to highly robust results in terms of non arbitrary identified bull and bear markets.

At this point the IBB algorithm is already completed and uses only one variable - namely the window size $F$ or $f$, respectively. The algorithms by Pagan and Sossounov (2003) and by Bry and Boschan (1971) require further steps during their execution. These steps are 1) testing if the identified market phases and cycles achieve the postulated minimum duration or minimum price variation, 2) eliminating (if necessary) market phases and cycles which do not achieve these postulations and 3) eliminating local maxima and minima which are too close to the start or the end of the entire time series. The first two steps do not have to be executed in the IBB algorithm due to the abandonment of minimum durations and minimum price variations. And the last of these steps does not have to be executed because the algorithm itself does not allow the detection of local maxima or minima which are too close to the start or the end of the entire time series.

As can be seen easily the IBB algorithm belongs to the semi-parametric rule-based methods. According to Kole and van Dijk (2017) these are better qualified for the retrospective identification of market phases than the parametric Markov switching models induced by Hamilton (1989). In contrast, Markov switching models are way better for forecasting market phase changes. But due to the consideration of returns as well as variances it can happen, for example, that in spite of increasing prices a market phase is retrospectively identified as a bear market due to a high variance, which can also occur during bull markets from time to time (Kole and van Dijk, 2017). The here used definition of bull (bear) markets solely focuses on increasing (decreasing) prices. In general, bull (bear) markets are associated in addition with positive (negative) returns and low (high) variances (see e.g. Chauvet and Potter, 2000; Kole and van Dijk, 2017; Maheu and McCurdy, 2000). As a matter of fact positive (negative) returns follow from increasing (decreasing) prices and so the IBB algorithm can also be applied to cumulative stock returns instead of stock prices without any changes in results. Moreover, bull markets are lasting longer than bear markets in general (see e.g. Chauvet and Potter, 2000; Gonzalez et al., 2006; Pagan and Sossounov, 2003).

Furthermore, the IBB algorithm is and should be applied to non smoothed time series like it is also done by Pagan and Sossounov (2003) and is not done so by Bry and Boschan (1971). Smoothing the time series would efface extreme prices, which might be most interesting. In addition, even a symmetric smoothing method would result into differing data points which are identified as the beginning of a market phase. So, not the highest (lowest) price would represent 
the beginning of a bull (bear) market, but some data point to the left or right of it. Besides, the choice of the smoothing method itself would affect the results, so that choosing a method would raise the arbitrariness of the results again.

\section{Application}

Due to the fact that bull (bear) markets describe increasing (decreasing) stock markets the IBB algorithm is here solely applied to diverse stock market indices. Of course every kind of stock market time series - not only indices - can be divided by it into bull and bear markets. So the IBB algorithm is not some kind of special case, which works only for the here selected time series.

\subsection{Data}

Although the IBB algorithm is especially made for the analysis of higher frequent data like daily prices it is applicable to lower frequent data as well. So beside daily data $(d d)$, weekly data $(w d)$ and monthly data $(m d)$ are also investigated here. The prices of all time series are closing prices - daily closing prices, weekly closing prices and monthly closing prices, respectively. The data used in the paper was obtained from Thomson Reuters Eikon and covers a period of more than 24 years from July 31, 1992 until September 30, 2016. For illustration, the data set consists of six stock market indices from five countries: Dow Jones and S\&P500 (USA each), Nikkei (Japan), DAX (Germany), FTSE (Great Britain) and SMI (Switzerland). All index prices are denominated in their respective local currency. Table 1 reports summary statistics of all six indices for daily, weekly and monthly data. The table contains for level prices the total number of observations and the average empirical number of observations per year. The latter lies for the respective indices between 246 and 253 observations p.a. for daily data and amounts to 52 observations p.a. for weekly data and 12 observations p.a. for monthly data for all six indices. Moreover table 1 contains the overall returns for the whole time span, which are computed as differences of the final and initial log prices. For returns - computed as first differences of log prices - the means as well as standard deviations and volatilities for the entire period of time are tabulated. Volatilities are calculated as square roots of the average number of observations p.a. times the standard deviations. So it represents some kind of annualized standard deviation. Furthermore, table 1 displays for which values of $f$ the most robust results occur and so which values of $f$ are 
finally used as the most robust ones in the IBB algorithm. Aside, the most robust range of values of $f$ are listed, too.

Table 1. Summary statistics

\begin{tabular}{|c|c|c|c|c|c|c|c|c|}
\hline \multirow[b]{2}{*}{$\begin{array}{l}\text { stock market } \\
\text { indices }\end{array}$} & \multicolumn{2}{|c|}{ no. of observ. } & \multirow[b]{2}{*}{$\begin{array}{l}\text { overall } \\
\text { return }\end{array}$} & \multirow[b]{2}{*}{$\begin{array}{l}\text { mean } \\
\text { return }\end{array}$} & \multirow[b]{2}{*}{$\begin{array}{l}\text { standard } \\
\text { deviation }\end{array}$} & \multirow[b]{2}{*}{ volatility } & \multicolumn{2}{|c|}{ most robust } \\
\hline & total & p.a. & & & & & range & $f$ \\
\hline DowJones $_{d d}$ & 6089 & 252 & $168.54 \%$ & $0.0277 \%$ & $1.0924 \%$ & $17.34 \%$ & 145 to 621 & 383 \\
\hline $\mathrm{S} \& \mathrm{P} 500_{d d}$ & 6089 & 252 & $163.15 \%$ & $0.0268 \%$ & $1.1525 \%$ & $18.29 \%$ & 143 to 685 & 414 \\
\hline Nikkei $_{d d}$ & 5942 & 246 & $3.34 \%$ & $0.0006 \%$ & $1.5167 \%$ & $23.79 \%$ & 354 to 676 & 515 \\
\hline $\operatorname{DAX}_{d d}$ & 6124 & 253 & $187.71 \%$ & $0.0307 \%$ & $1.4596 \%$ & $23.22 \%$ & 207 to 376 & 291 \\
\hline $\mathrm{FTSE}_{d d}$ & 6108 & 253 & $105.61 \%$ & $0.0173 \%$ & $1.1420 \%$ & $18.16 \%$ & 162 to 363 & 262 \\
\hline $\mathrm{SMI}_{d d}$ & 6079 & 251 & $150.99 \%$ & $0.0248 \%$ & $1.1746 \%$ & $18.61 \%$ & 294 to 745 & 519 \\
\hline DowJones $_{w d}$ & 1262 & 52 & $168.54 \%$ & $0.1337 \%$ & $2.2601 \%$ & $16.30 \%$ & 31 to 128 & 79 \\
\hline $\mathrm{S} \& \mathrm{P} 500_{w d}$ & 1262 & 52 & $163.15 \%$ & $0.1294 \%$ & $2.3250 \%$ & $16.77 \%$ & 29 to 141 & 85 \\
\hline Nikkei $_{w d}$ & 1262 & 52 & $3.34 \%$ & $0.0026 \%$ & $3.0585 \%$ & $22.05 \%$ & 83 to 143 & 113 \\
\hline $\mathrm{DAX}_{w d}$ & 1262 & 52 & $187.71 \%$ & $0.1489 \%$ & $3.1275 \%$ & $22.55 \%$ & 42 to 77 & 59 \\
\hline $\mathrm{FTSE}_{w d}$ & 1262 & 52 & $105.61 \%$ & $0.0838 \%$ & $2.3516 \%$ & $16.96 \%$ & 78 to 156 & 117 \\
\hline $\mathrm{SMI}_{w d}$ & 1262 & 52 & $150.99 \%$ & $0.1197 \%$ & $2.5916 \%$ & $18.69 \%$ & 78 to 154 & 116 \\
\hline DowJones $_{m d}$ & 291 & 12 & $168.54 \%$ & $0.5812 \%$ & $4.1249 \%$ & $14.29 \%$ & 9 to 24 & 16 \\
\hline $\mathrm{S} \& \mathrm{P} 500_{m d}$ & 291 & 12 & $163.15 \%$ & $0.5626 \%$ & $4.1846 \%$ & $14.50 \%$ & 7 to 32 & 19 \\
\hline Nikkei $_{m d}$ & 291 & 12 & $3.34 \%$ & $0.0115 \%$ & $5.9056 \%$ & $20.46 \%$ & 19 to 34 & 26 \\
\hline $\operatorname{DAX}_{m d}$ & 291 & 12 & $187.71 \%$ & $0.6473 \%$ & $6.1536 \%$ & $21.32 \%$ & 10 to 18 & 14 \\
\hline FTSE $_{m d}$ & 291 & 12 & $105.61 \%$ & $0.3642 \%$ & $3.9804 \%$ & $13.79 \%$ & 17 to 35 & 26 \\
\hline $\mathrm{SMI}_{m d}$ & 291 & 12 & $150.99 \%$ & $0.5207 \%$ & $4.5175 \%$ & $15.65 \%$ & 15 to 34 & 24 \\
\hline
\end{tabular}

Note: The table contains summary statistics for six indices from five countries in three frequencies. The six indices are: DowJones and S\&P500 (USA each), Nikkei (Japan), DAX (Germany), FTSE (Great Britain) and SMI (Switzerland). The data are closing prices and cover the period from July 31, 1992 until September 30, 2016. The upper block contains daily data $(d d)$, the middle block weekly data $(w d)$ and the lower block monthly data $(m d)$. The table lists for level prices the total number of observations (no. of. observ.) and number of observations per year (p.a.), the most robust range of $f$ and the most robust $f$. Furthermore, the table states for returns the overall and mean return, the standard deviation and volatility.

It emerges that all most robust $f$ comprise at least one year, which leads to a total window size $F$ of at least two years. In some cases $f$ exceeds even two years and $F$ therefore even four years. This underlines the ambition to identify rather longer subperiods than shorter ones. Looking at the most robust range it can be seen that the results are relatively high robust against changes of the only variable of the IBB algorithm. This can be emphasized by looking at the percentage variation of the most robust $f$ inside the most robust range. For example, the most robust $f$ for the weekly data of the Nikkei is 113 and the most robust range goes from 83 to 143 . This means that even an $f$ which is $26.55 \%$ smaller or larger than the most robust one would still yield the same results. For every other index the respective most robust range is even larger than for this example and so the percentage variation is larger as well. The highest percentage variation can be found for the monthly data of the S\&P500. There the most robust $f$ is 19 and even for $f=32$ the results would be still the same. This corresponds to a percentage variation of $68.42 \%$.

Looking at the overall returns and mean returns the Nikkei performs worst over the entire period of time - independently of the frequency. Besides, it exhibits the highest standard deviation and volatility for daily data and the second highest for weekly and monthly data. For the latter, only the DAX has higher standard deviations and volatilities, but exhibits instead the highest overall 
return and mean return for all kinds of frequencies. By comparison the other four indices have conspicuously lower standard deviations and volatilities, with the differences between the indices being rather small. The returns of the Dow Jones, S\&P500 and SMI are as well somewhat similar. Solely the returns of the FTSE are slightly lower, but are far from being as low as the ones of the Nikkei.
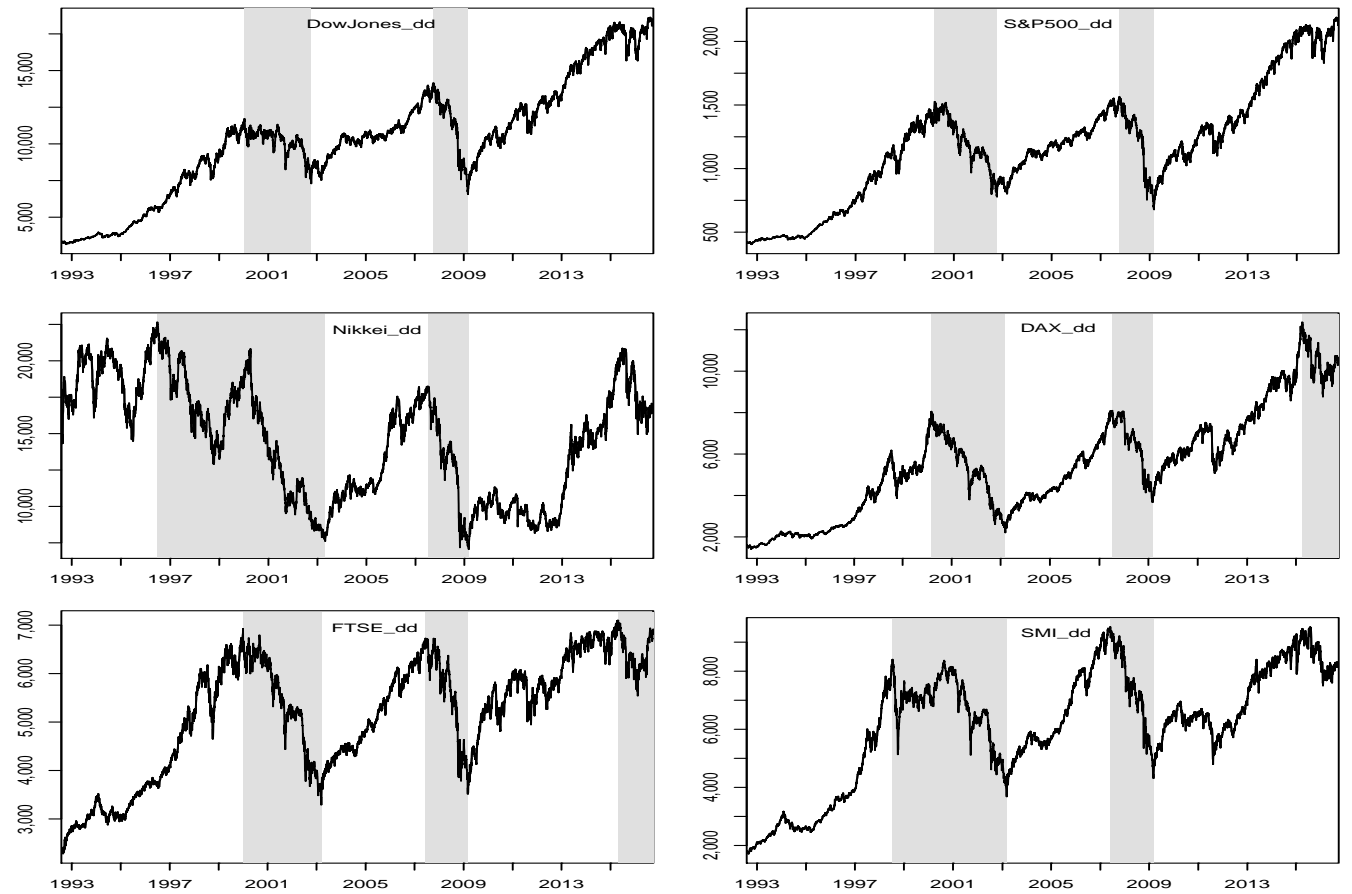

Figure 4. Identified bull and bear markets for daily closing prices. The figure reports daily closing prices of six indices from five countries. The six indices are: DowJones and S\&P500 (USA each), Nikkei (Japan), DAX (Germany), FTSE (Great Britain) and SMI (Switzerland). The time series comprise the period from July 31, 1992 until September 30, 2016. The shaded areas represent the bear markets which are identified by the IBB algorithm. The non shaded areas represent the identified bull markets.

This can also be seen looking at the figures $4-6^{1}$, which illustrate for the six indices the daily, weekly and monthly closing prices, respectively. While the Dow Jones and S\&P500 show quite similar curves, the three European indices exhibit as well curves, which are quite similar to each other. Even the two American and three European indices show curves which seem to be somewhat related. Only the Nikkei exhibits a more differing curve, which is what makes it so interesting to look at it beside the other indices and to show the applicability of the IBB algorithm to all stock market indices. As can be seen, all indices feature a lot of ups and downs and every index exhibits longer periods of time for which the prices are overall increasing or decreasing in

1 For illustration purposes figure 4 displays results for daily closing prices. See Appendix A for results for weekly closing prices (figure 5) and monthly closing prices (figure 6). 
spite of little countermovements. These (sub-) periods of time represent bull and bear markets and are of special interest for longer term investments in stock markets. Now, these subperiods have to be identified properly.

\subsection{Results}

In tables $2-4^{2}$ all identified market phases for the six indices and three frequencies, which result from the application of the IBB algorithm, are tabulated chronologically each. For every time series the beginning of the first market phase and the end of the last market phase are not given by an actual turn from one market phase to the other, but by the available data. All identified market phases of tables 2 - 4 follow directly from the identified possible beginnings of bull (bear) markets, which result from the application of the functions $\operatorname{Max}(i)(\operatorname{Min}(i))$ and $L_{i}^{\operatorname{Max}}\left(L_{i}^{\operatorname{Min}}\right)$ by using the most robust $f$. Using the most robust $f$ here leads to already alternating bull and bear markets, so that all possible beginnings are already actual ones. So the elimination of successive possible beginnings of the same market phase is not necessary in any of the analyzed time series. This makes the IBB algorithm even easier to work with. But it has to be mentioned that this is not necessarily the case for every window size and not even for every window size which is within the most robust range for which the overall results stay the same. So there are window sizes for which the elimination algorithm is needed. However, it is possible that for every kind of stock market time series the use of the most robust $f$, leads to already alternating bull and bear markets, so that there is no need for the elimination algorithm. To give a final answer to this issue further research for this special question has to be done.

The tables 2 - 4 contain for every identified market phase the data points $i$ on which the market phases begin and end as well as their corresponding date. Additionally, for level prices the total number of observations and for returns the overall returns, mean returns, standard deviations and volatilities for every market phase are listed. The time series are divided by the IBB algorithm in five to six market phases consisting of three bull markets and two to three bear markets. All bull (bear) markets have the typical positive (negative) overall and mean returns, whereas in general the bull (bear) markets exhibit the typical low (high) standard deviations and volatilites. Deviations from this occur only in a few noteworthy cases. So, for example, the last bull market of the

2 For illustration purposes table 2 represents results for daily closing prices. See Appendix B for results for weekly closing prices (table 3 ) and monthly closing prices (table 4). 
Table 2. Identified bull and bear markets for daily data

\begin{tabular}{|c|c|c|c|c|c|c|c|c|c|c|}
\hline \multirow[b]{2}{*}{$\begin{array}{l}\text { stock market } \\
\text { indices }\end{array}$} & \multirow[b]{2}{*}{$\begin{array}{l}\text { market } \\
\text { phase }\end{array}$} & \multicolumn{2}{|c|}{ beginning } & \multicolumn{2}{|r|}{ end } & \multirow[b]{2}{*}{$\begin{array}{l}\text { no. of } \\
\text { observ. }\end{array}$} & \multirow[b]{2}{*}{$\begin{array}{l}\text { overall } \\
\text { return }\end{array}$} & \multirow[b]{2}{*}{$\begin{array}{l}\text { mean } \\
\text { return }\end{array}$} & \multirow[b]{2}{*}{$\begin{array}{l}\text { standard } \\
\text { deviation }\end{array}$} & \multirow[b]{2}{*}{ volatility } \\
\hline & & $i$ & date & $i$ & date & & & & & \\
\hline \multirow[t]{5}{*}{ DowJones $_{d d}$} & bull & 1 & 07.31 .1992 & 1885 & 01.14 .2000 & 1885 & $+123.96 \%$ & $+0.0658 \%$ & $0.8881 \%$ & $14.10 \%$ \\
\hline & bear & 1885 & 01.14 .2000 & 2570 & 10.09 .2002 & 686 & $-47.56 \%$ & $-0.0676 \%$ & $1.4053 \%$ & $22.31 \%$ \\
\hline & bull & 2570 & 10.09 .2002 & 3828 & 10.09 .2007 & 1259 & $+66.47 \%$ & $+0.0505 \%$ & $0.8377 \%$ & $13.30 \%$ \\
\hline & bear & 3828 & 10.09 .2007 & 4183 & 03.09.2009 & 356 & $-77.17 \%$ & $-0.2144 \%$ & $2.1886 \%$ & $34.74 \%$ \\
\hline & bull & 4183 & 03.09.2009 & 6089 & 09.30 .2016 & 1900 & $+102.83 \%$ & $+0.0533 \%$ & $0.9760 \%$ & $15.49 \%$ \\
\hline \multirow{5}{*}{$\mathrm{S} \& \mathrm{P} 500_{d d}$} & bull & 1 & 07.31 .1992 & 1933 & 03.24 .2000 & 1933 & $+128.11 \%$ & $+0.0663 \%$ & $0.9150 \%$ & $14.53 \%$ \\
\hline & bear & 1933 & 03.24 .2000 & 2570 & 10.09 .2002 & 638 & $-67.62 \%$ & $-0.1060 \%$ & $1.4428 \%$ & $22.90 \%$ \\
\hline & bull & 2570 & 10.09 .2002 & 3828 & 10.09 .2007 & 1259 & $+70.06 \%$ & $+0.0535 \%$ & $0.8587 \%$ & $13.63 \%$ \\
\hline & bear & 3828 & 10.09 .2007 & 4183 & 03.09.2009 & 356 & $-83.88 \%$ & $-0.2333 \%$ & $2.3925 \%$ & $37.98 \%$ \\
\hline & bull & 4183 & 03.09 .2009 & 6089 & 09.30 .2016 & 1907 & $+116.47 \%$ & $+0.0605 \%$ & $1.0599 \%$ & $16.83 \%$ \\
\hline \multirow[t]{5}{*}{ Nikkei $_{d d}$} & bull & 1 & 07.31 .1992 & 965 & 06.26 .1996 & 965 & $+35.39 \%$ & $+0.0367 \%$ & $0.0367 \%$ & $20.54 \%$ \\
\hline & bear & 965 & 06.26 .1996 & 2648 & 04.28 .2003 & 1684 & $-109.17 \%$ & $-0.0646 \%$ & $1.5724 \%$ & $24.66 \%$ \\
\hline & bull & 2648 & 04.28 .2003 & 3681 & 07.09.2007 & 1034 & $+87.56 \%$ & $+0.0835 \%$ & $1.1475 \%$ & $18.00 \%$ \\
\hline & bear & 3681 & 07.09.2007 & 4089 & 03.10 .2009 & 409 & $-95.11 \%$ & $-0.2309 \%$ & $2.4895 \%$ & $39.05 \%$ \\
\hline & bull & 4089 & 03.10 .2009 & 5942 & 09.30 .2016 & 1854 & $+84.66 \%$ & $+1.4531 \%$ & $1.4531 \%$ & $22.79 \%$ \\
\hline \multirow[t]{6}{*}{$\operatorname{DAX}_{d d}$} & bull & 1 & 07.31 .1992 & 1912 & 03.07 .2000 & 1912 & $+161.22 \%$ & $+0.0844 \%$ & $1,2619 \%$ & $20,07 \%$ \\
\hline & bear & 1912 & 03.07 .2000 & 2675 & 03.12 .2003 & 764 & $-129.77 \%$ & $-0.1684 \%$ & $2.0410 \%$ & $32.46 \%$ \\
\hline & bull & 2675 & 03.12 .2003 & 3783 & 07.16 .2007 & 1109 & $+130.28 \%$ & $+0.1134 \%$ & $1.1404 \%$ & $18.14 \%$ \\
\hline & bear & 3783 & 07.16 .2007 & 4199 & 03.06.2009 & 417 & $-79.34 \%$ & $-0.1899 \%$ & $2.0862 \%$ & $33.18 \%$ \\
\hline & bull & 4199 & 03.06.2009 & 5748 & 04.10 .2015 & 1550 & $+121.64 \&$ & $+0.0780 \%$ & $1.3082 \%$ & $20.81 \%$ \\
\hline & bear & 5748 & 04.10 .2015 & 6124 & 09.30 .2016 & 377 & $-16.32 \%$ & $-0.0388 \%$ & $1.5158 \%$ & $24.11 \%$ \\
\hline \multirow[t]{6}{*}{$\operatorname{FTSE}_{d d}$} & bull & 1 & 07.31 .1992 & 1875 & 12.30 .1999 & 1875 & $+106.06 \%$ & $+0.0566 \%$ & $0.9227 \%$ & $14.68 \%$ \\
\hline & bear & 1875 & 12.30 .1999 & 2682 & 03.12 .2003 & 808 & $-74.59 \%$ & $-0.0906 \%$ & $1.4628 \%$ & $23.27 \%$ \\
\hline & bull & 2682 & 03.12 .2003 & 3758 & 06.15 .2007 & 1077 & $+76.69 \%$ & $+0.0620 \%$ & $0.7883 \%$ & $12.54 \%$ \\
\hline & bear & 3758 & 06.15 .2007 & 4193 & 03.03.2009 & 435 & $-65.07 \%$ & $-0.1464 \%$ & $2.0368 \%$ & $32.40 \%$ \\
\hline & bull & 4193 & 03.03.2009 & 5745 & 04.27 .2015 & 1553 & $+70.44 \%$ & $+0.0433 \%$ & $1.0401 \%$ & $16.54 \%$ \\
\hline & bear & 5745 & 04.27 .2015 & 6108 & 09.30 .2016 & 364 & $-2.92 \%$ & $-0.0067 \%$ & $1.1636 \%$ & $18.51 \%$ \\
\hline \multirow[t]{5}{*}{$\mathrm{SMI}_{d d}$} & bull & 1 & 07.31 .1992 & 1499 & 07.21 .1998 & 1499 & $+154.29 \%$ & $+0.1030 \%$ & $0.9298 \%$ & $14.73 \%$ \\
\hline & bear & 1499 & 07.21 .1998 & 2670 & 03.12 .2003 & 1172 & $-82.80 \%$ & $-0.0705 \%$ & $1.5138 \%$ & $23.98 \%$ \\
\hline & bull & 2670 & 03.12 .2003 & 3734 & 06.01 .2007 & 1064 & $+95.29 \%$ & $+0.0884 \%$ & $0.8695 \%$ & $13.78 \%$ \\
\hline & bear & 3734 & 06.01 .2007 & 4177 & 03.09.2009 & 444 & $-79.42 \%$ & $-0.1770 \%$ & $1.8544 \%$ & $29.38 \%$ \\
\hline & bull & 4177 & 03.09.2009 & 6029 & 09.30 .2016 & 1853 & $+63.63 \%$ & $+0.0334 \%$ & $1.0335 \%$ & $16.27 \%$ \\
\hline
\end{tabular}

Note: The table contains detailed information about the identified bull and bear markets for six indices from five countries. The six indices are: DowJones and S\&P500 (USA each), Nikkei (Japan), DAX (Germany), FTSE (Great Britain) and SMI (Switzerland). The data are daily closing prices and cover the period from July 31, 1992 until September 30, 2016. The table lists the identified market phases as well as the beginning and end of the respective market phase in form of the data point $i$ and the corresponding date. The date is indicated in the date format mm.dd.yyyy. For every market phase the number of observations (no. of. observ.) in level prices as well as the overall and mean return, the standard deviation and volatility in returns are added.

Nikkei $_{d d}$ and the Nikkei ${ }_{w d}$ has slightly higher volatility and the last bear market of the $\mathrm{SMI}_{d d}$ has slightly lower volatility than usual. Here, for example, the parametric Markov switching models could reach their limits considering both returns and variances. With little exceptions the bull markets last for considerably longer than the bear markets as expected. Only the first bear market of the Nikkei in all frequencies and the first bear market of the $\mathrm{SMI}_{d d}$ and $\mathrm{SMI}_{m d}$ last somewhat longer than usual.

This can also be seen in figures $4-6$, which show besides the curves of all indices and frequencies the identified bull and bear markets. Those are illustrated by different shadings. The non shaded areas represent bull markets and the shaded ones bear markets. In most cases - for example, looking at the results for the S\&P500 and the FTSE - the identified market phases meet easily the market phases which most people would possibly identify by their intuition. But in some cases the identified market phases are maybe not that intuitive. Especially the identified first bear market of the Nikkei would possibly be subdivided in two bear and one bull market by intuition. But the resulting bull market would only last for 370, 80 or 19 observations for daily, weekly or monthly data, 
respectively, and so it would last for a considerably shorter period of time in comparison to all the other identified bull markets. That is why the IBB algorithm does not detect this bull market, but interprets it instead as a countermovement only. Hence, the IBB algorithm is neither restricted by intuitive views nor affected by arbitrary choices. At the same time the identified market phases are always longer lasting, which therefore can be used for longer term investment decisions.

For the six indices and three frequencies all respectively identified first bear markets comprise at least the time period from August 31, 2000 until September 30, 2002 and the second bear markets comprise at least the time period from December 28, 2007 until February 27, 2009. Hence, independently of the index and the frequency these periods of time exhibit decreasing stock market prices in American, European and Asian stock markets. So, thanks to the IBB algorithm and the resulting subperiods the time series can be analyzed in more detail to find out how different assets behave in different (sub-) periods of time and if and when relations exist between them.

\section{Conclusion}

The present paper gives a description of the IBB algorithm to identify bull and bear markets and thus to divide time series into subperiods. It can be applied to every kind of stock market time series and frequency. In addition, it can even be applied to non stock market time series to detect local maxima and minima and therefore turning points between increasing and decreasing values. The partition by the IBB algorithm occurs under the ambition to find rather longer than shorter subperiods and to avoid the use of arbitrary assumptions or constraints. The IBB algorithm is therefore distinct from existing approaches. Such approaches always postulate exogenously that a market phase can only be identified as a separate market phase if it lasts for at least a certain amount of time or if the prices increase or decrease for at least a certain percentage. But there is no general agreement what 'certain' actually means. Because the IBB algorithm does not postulate exogenously a minimum duration or a minimum price variation, it is considerably less arbitrary. Another circumstance that makes the IBB algorithm less arbitrary is the application of it to non smoothed time series, whereby a smoothing method does not have to be chosen. All this makes it very easy to execute it and makes the computational burden comparatively low. At the same time the whole IBB algorithm only needs one variable: The window size for which local maxima and minima have to be identified. But even this does not have to be chosen arbitrarily, because it 
is endogenously determined by the most robust window size. So, for example for the time series which are analyzed in the present paper, the window size can be varied from around $27 \%$ up to around $68 \%$ without any change in the resulting market phases, which are identified by the IBB algorithm. This makes the approach a pretty robust one. Furthermore, the typical characteristics of bull (bear) markets - positive (negative) returns, low (high) volatility and longer lasting bull than bear markets - are found in general. Knowing the bull and bear markets fosters further analyses, like investigating correlations between different assets in different (sub-) periods of time.

\section{Appendices}

\section{A Figures}
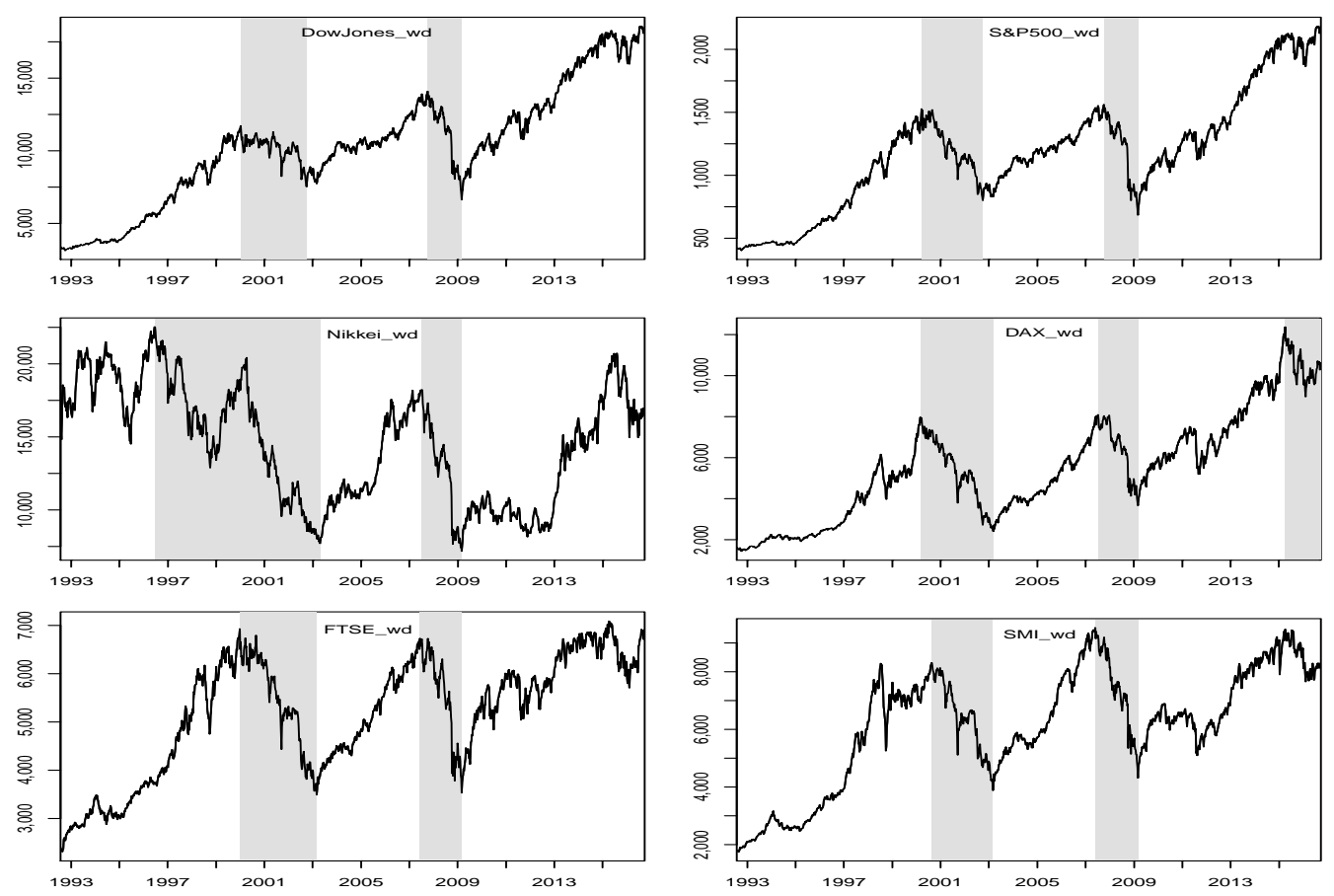

Figure 5. Identified bull and bear markets for weekly closing prices. The figure reports weekly closing prices of six indices from five countries. The six indices are: DowJones and S\&P500 (USA each), Nikkei (Japan), DAX (Germany), FTSE (Great Britain) and SMI (Switzerland). The time series comprise the period from July 31, 1992 until September 30, 2016. The shaded areas represent the bear markets which are identified by the IBB algorithm. The non shaded areas represent the identified bull markets. 

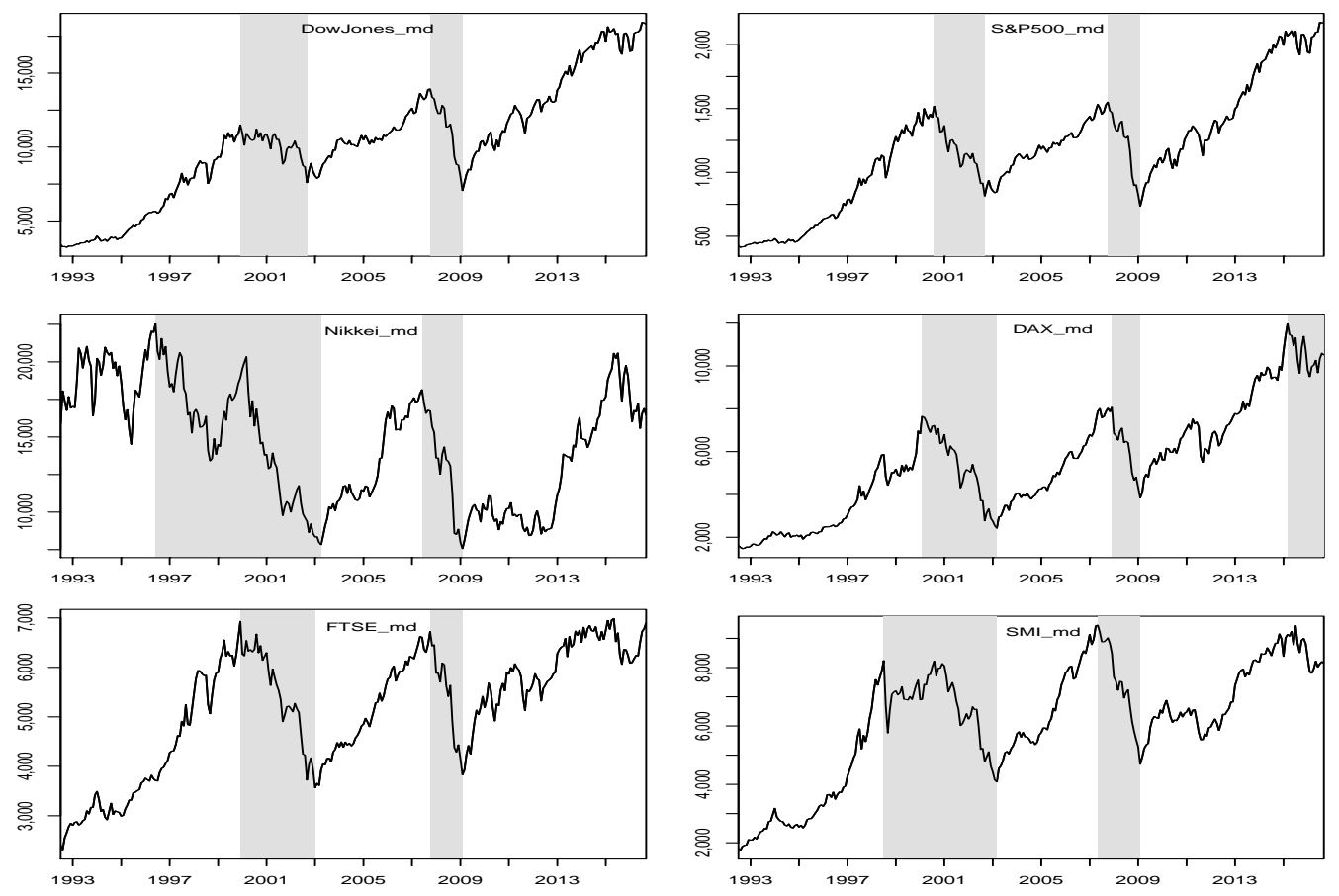

Figure 6. Identified bull and bear markets for monthly closing prices. The figure reports monthly closing prices of six indices from five countries. The six indices are: DowJones and S\&P500 (USA each), Nikkei (Japan), DAX (Germany), FTSE (Great Britain) and SMI (Switzerland). The time series comprise the period from July 31, 1992 until September 30, 2016. The shaded areas represent the bear markets which are identified by the IBB algorithm. The non shaded areas represent the identified bull markets. 


\section{B Tables}

Table 3. Identified bull and bear markets for weekly data

\begin{tabular}{|c|c|c|c|c|c|c|c|c|c|c|}
\hline \multirow[b]{2}{*}{$\begin{array}{l}\text { stock market } \\
\text { indices }\end{array}$} & \multirow[b]{2}{*}{$\begin{array}{c}\text { market } \\
\text { phase }\end{array}$} & \multicolumn{2}{|c|}{ beginning } & \multicolumn{2}{|r|}{ end } & \multirow[b]{2}{*}{$\begin{array}{l}\text { no. of } \\
\text { observ. }\end{array}$} & \multirow[b]{2}{*}{$\begin{array}{l}\text { overall } \\
\text { return }\end{array}$} & \multirow[b]{2}{*}{$\begin{array}{l}\text { mean } \\
\text { return }\end{array}$} & \multirow[b]{2}{*}{$\begin{array}{l}\text { standard } \\
\text { deviation }\end{array}$} & \multirow[b]{2}{*}{ volatility } \\
\hline & & $i$ & date & $i$ & date & & & & & \\
\hline \multirow[t]{5}{*}{ DowJones $_{w d}$} & bull & 1 & 07.31 .1992 & 390 & 01.14 .2000 & 390 & $+123.96 \%$ & $+0.3187 \%$ & $1.8667 \%$ & $13.46 \%$ \\
\hline & bear & 390 & 01.14 .2000 & 532 & 10.04 .2002 & 143 & $-44.29 \%$ & $-0.2976 \%$ & $3.0492 \%$ & $21.99 \%$ \\
\hline & bull & 532 & 10.04 .2002 & 794 & 10.12.2007 & 262 & $+62.70 \%$ & $+0.2298 \%$ & $1.7109 \%$ & $12.34 \%$ \\
\hline & bear & 794 & 10.12 .2007 & 867 & 03.08.2009 & 74 & $-75.45 \%$ & $-1.0171 \%$ & $4.1135 \%$ & $29.66 \%$ \\
\hline & bull & 867 & 03.08.2009 & 1262 & 09.30 .2016 & 396 & $+101.62 \%$ & $+0.2405 \%$ & $2.0352 \%$ & $14.68 \%$ \\
\hline \multirow[t]{5}{*}{ S\&P500 ${ }_{w d}$} & bull & 1 & 07.31 .1992 & 400 & 03.24 .2000 & 400 & $+128.11 \%$ & $+0.3211 \%$ & $1.9153 \%$ & $13.81 \%$ \\
\hline & bear & 400 & 03.24 .2000 & 532 & 10.04 .2002 & 133 & $-64.60 \%$ & $-0.4541 \%$ & $3.0008 \%$ & $21.64 \%$ \\
\hline & bull & 532 & 10.04 .2002 & 794 & 10.12 .2007 & 263 & $+66.83 \%$ & $+0.2416 \%$ & $1.6811 \%$ & $12.12 \%$ \\
\hline & bear & 794 & 10.12 .2007 & 867 & 03.06.2009 & 73 & $-82.65 \%$ & $-1.1133 \%$ & $4.3972 \%$ & $31.71 \%$ \\
\hline & bull & 867 & 03.06.2009 & 1262 & 09.30 .2016 & 395 & $+115.46 \%$ & $+0.2732 \%$ & $2.1783 \%$ & $15.71 \%$ \\
\hline \multirow[t]{5}{*}{ Nikkei $_{w d}$} & bull & 1 & 07.31 .1992 & 205 & 06.28 .1996 & 205 & $+34.79 \%$ & $+0.1705 \%$ & $2.8085 \%$ & $20.25 \%$ \\
\hline & bear & 205 & 06.28 .1996 & 561 & 04.25 .2003 & 357 & $-107.37 \%$ & $-0.3008 \%$ & $3.0885 \%$ & $22.27 \%$ \\
\hline & bull & 561 & 04.25 .2003 & 781 & 07.13 .2007 & 221 & $+86.24 \%$ & $+0.3801 \%$ & $2.2655 \%$ & $16.24 \%$ \\
\hline & bear & 781 & 07.13 .2007 & 867 & 03.06.2009 & 87 & $-93.32 \%$ & $-1.0665 \%$ & $4.8318 \%$ & $34.84 \%$ \\
\hline & bull & 867 & 03.06.2009 & 1262 & 09.30 .2016 & 395 & $+83.00 \%$ & $+0.1960 \%$ & $2.9582 \%$ & $21.33 \%$ \\
\hline \multirow[t]{6}{*}{$\mathrm{DAX}_{w d}$} & bull & 1 & 07.31 .1992 & 398 & 03.10 .2000 & 398 & $+160.11 \%$ & $+0.4033 \%$ & $2.6775 \%$ & $19.31 \%$ \\
\hline & bear & 398 & 03.10 .2000 & 555 & 03.14 .2003 & 158 & $-119.96 \%$ & $-0.7580 \%$ & $4.0056 \%$ & $28.88 \%$ \\
\hline & bull & 555 & 03.14 .2003 & 781 & 07.13 .2007 & 227 & $+121.42 \%$ & $+0.5297 \%$ & $2.4237 \%$ & $17.48 \%$ \\
\hline & bear & 781 & 07.13 .2007 & 867 & 03.06.2009 & 87 & $-79.18 \%$ & $-0.9037 \%$ & $4.9293 \%$ & $35.55 \%$ \\
\hline & bull & 867 & 03.06.2009 & 1185 & 04.10 .2015 & 319 & $+121.64 \%$ & $+0.3665 \%$ & $2.8116 \%$ & $20.27 \%$ \\
\hline & bear & 1185 & 04.10 .2015 & 1262 & 09.30 .2016 & 78 & $-16.32 \%$ & $-0.1664 \%$ & $3.0209 \%$ & $21.78 \%$ \\
\hline \multirow[t]{5}{*}{$\mathrm{FTSE}_{w d}$} & bull & 1 & 07.31 .1992 & 388 & 12.30 .1999 & 388 & $+106.06 \%$ & $+0.2741 \%$ & $1.9686 \%$ & $14.20 \%$ \\
\hline & bear & 388 & 12.30 .1999 & 554 & 03.07.2003 & 167 & $-68.55 \%$ & $-0.3997 \%$ & $2.6945 \%$ & $19.43 \%$ \\
\hline & bull & 554 & 03.07.2003 & 777 & 06.15 .2007 & 224 & $+65.66 \%$ & $+0.2726 \%$ & $1.5668 \%$ & $11.30 \%$ \\
\hline & bear & 777 & 06.15.2007 & 867 & 03.06 .2009 & 91 & $-64.54 \%$ & $-0.6715 \%$ & $4.3830 \%$ & $31.61 \%$ \\
\hline & bull & 867 & 03.06.2009 & 1262 & 09.30 .2016 & 395 & $+66.99 \%$ & $+0.1486 \%$ & $2.2200 \%$ & $16.01 \%$ \\
\hline \multirow[t]{5}{*}{$\mathrm{SMI}_{w d}$} & bull & 1 & 07.31 .1992 & 422 & 08.25 .2000 & 422 & $+153.09 \%$ & $+0.3636 \%$ & $2.3616 \%$ & $17.03 \%$ \\
\hline & bear & 422 & 08.25 .2000 & 554 & 03.07.2003 & 133 & $-76.18 \%$ & $-0.5673 \%$ & $3.3815 \%$ & $24.38 \%$ \\
\hline & bull & 554 & 03.07.2003 & 775 & 06.01 .2007 & 222 & $+89.87 \%$ & $+0.3747 \%$ & $1.8042 \%$ & $13.01 \%$ \\
\hline & bear & 775 & 06.01.2007 & 867 & 03.06.2009 & 93 & $-79.33 \%$ & $-0.8359 \%$ & $4.4307 \%$ & $31.95 \%$ \\
\hline & bull & 867 & 03.06 .2009 & 1262 & 09.30.2016 & 396 & $+63.54 \%$ & $+0.1392 \%$ & $2.2396 \&$ & $16.15 \%$ \\
\hline
\end{tabular}

Note: The table contains detailed information about the identified bull and bear markets for six indices from five countries. The six indices are: DowJones and S\&P500 (USA each), Nikkei (Japan), DAX (Germany), FTSE (Great Britain) and SMI (Switzerland). The data are weekly closing prices and cover the period from July 31, 1992 until September 30, 2016. The table lists the identified market phases as well as the beginning and end of the respective market phase in form of the data point $i$ and the corresponding date. The date is indicated in the date format mm.dd.yyyy. For every market phase the number of observations (no. of. observ.) in level prices as well as the overall and mean return, the standard deviation and volatility in returns are added. 
Table 4. Identified bull and bear markets for monthly data

\begin{tabular}{|c|c|c|c|c|c|c|c|c|c|c|}
\hline \multirow[b]{2}{*}{$\begin{array}{l}\text { stock market } \\
\text { indices }\end{array}$} & \multirow[b]{2}{*}{$\begin{array}{l}\text { market } \\
\text { phase }\end{array}$} & \multicolumn{2}{|c|}{ beginning } & \multicolumn{2}{|r|}{ end } & \multirow[b]{2}{*}{$\begin{array}{l}\text { no. of } \\
\text { observ. }\end{array}$} & \multirow[b]{2}{*}{$\begin{array}{l}\text { overall } \\
\text { return }\end{array}$} & \multirow[b]{2}{*}{$\begin{array}{l}\text { mean } \\
\text { return }\end{array}$} & \multirow[b]{2}{*}{$\begin{array}{l}\text { standard } \\
\text { deviation }\end{array}$} & \multirow[b]{2}{*}{ volatility } \\
\hline & & $i$ & date & $i$ & date & & & & & \\
\hline \multirow[t]{5}{*}{ DowJones $_{m d}$} & bull & 1 & 07.31 .1992 & 90 & 12.31.1999 & 90 & $+122.02 \%$ & $+1.3710 \%$ & $3.9081 \%$ & $13.54 \%$ \\
\hline & bear & 90 & 12.31 .1999 & 123 & 09.30.2002 & 34 & $-41.50 \%$ & $-1.0578 \%$ & $5.2336 \%$ & $18.13 \%$ \\
\hline & bull & 123 & 09.30 .2002 & 184 & 10.31 .2007 & 62 & $+60.70 \%$ & $+0.7660 \%$ & $3.3948 \%$ & $11.76 \%$ \\
\hline & bear & 184 & 10.31 .2007 & 200 & 02.27 .2009 & 17 & $-67.92 \%$ & $-3.9807 \%$ & $5.1950 \%$ & $18.00 \%$ \\
\hline & bull & 200 & 02.27 .2009 & 291 & 09.30 .2016 & 92 & $+95.25 \%$ & $+0.8998 \%$ & $3.8213 \%$ & $13.24 \%$ \\
\hline \multirow[t]{5}{*}{$\mathrm{S} \& \mathrm{P} 500_{m d}$} & bull & 1 & 07.31 .1992 & 98 & 08.31 .2000 & 98 & $+127.47 \%$ & $+1.3141 \%$ & $3.7474 \%$ & $12.98 \%$ \\
\hline & bear & 98 & 08.31 .2000 & 123 & 09.30 .2002 & 26 & $-62.14 \%$ & $-2.1634 \%$ & $5.2606 \%$ & $18.22 \%$ \\
\hline & bull & 123 & 09.30.2002 & 184 & 10.31 .2007 & 62 & $+64.21 \%$ & $+0.8476 \%$ & $3.1314 \%$ & $10.85 \%$ \\
\hline & bear & 184 & 10.31 .2007 & 200 & 02.27 .2009 & 17 & $-74.56 \%$ & $-4.2994 \%$ & $5.8398 \%$ & $20.23 \%$ \\
\hline & bull & 200 & 02.27 .2009 & 291 & 09.30 .2016 & 92 & $+108.17 \%$ & $+1.0492 \%$ & $3.9833 \%$ & $13.80 \%$ \\
\hline \multirow[t]{5}{*}{ Nikkei $_{m d}$} & bull & 1 & 07.31 .1992 & 48 & 06.28 .1996 & 48 & $+34.79 \%$ & $+0.7402 \%$ & $6.4139 \%$ & $22.22 \%$ \\
\hline & bear & 48 & 06.28 .1996 & 130 & 04.30 .2003 & 83 & $-105.67 \%$ & $-1.2421 \%$ & $5.7693 \%$ & $19.99 \%$ \\
\hline & bull & 130 & 04.30 .2003 & 180 & 06.29 .2007 & 51 & $+83.99 \%$ & $+1.6118 \%$ & $3.9213 \%$ & $13.58 \%$ \\
\hline & bear & 180 & 06.29 .2007 & 200 & 02.27 .2009 & 21 & $-87.40 \%$ & $-4.0927 \%$ & $7.6345 \%$ & $26.45 \%$ \\
\hline & bull & 200 & 02.27 .2009 & 291 & 09.30.2016 & 92 & $+77.63 \%$ & $+0.7844 \%$ & $5.4743 \%$ & $18.96 \%$ \\
\hline \multirow[t]{6}{*}{$\mathrm{DAX}_{m d}$} & bull & 1 & 07.31 .1992 & 92 & 02.29 .2000 & 92 & $+155.87 \%$ & $+1.7129 \%$ & $5.6775 \%$ & $19.67 \%$ \\
\hline & bear & 92 & 02.29 .2000 & 129 & 03.31 .2003 & 38 & $-114.86 \%$ & $-2.7284 \%$ & $8.4955 \%$ & $29.43 \%$ \\
\hline & bull & 129 & 03.31 .2003 & 186 & 12.28 .2007 & 58 & $+120.25 \%$ & $+1.9877 \%$ & $4.3104 \%$ & $14.93 \%$ \\
\hline & bear & 186 & 12.28 .2007 & 200 & 02.27 .2009 & 15 & $-74.14 \%$ & $-4.7779 \%$ & $6.9785 \%$ & $24.17 \%$ \\
\hline & bull & 200 & 02.27 .2009 & 273 & 03.31 .2015 & 74 & $+113.56 \%$ & $+1.3711 \%$ & $5.3065 \%$ & $18.38 \%$ \\
\hline & bear & 273 & 03.31 .2015 & 291 & 09.30.2016 & 19 & $-12.97 \%$ & $-0.4281 \%$ & $5.6050 \%$ & $19.42 \%$ \\
\hline \multirow[t]{5}{*}{$\mathrm{FTSE}_{m d}$} & bull & 1 & 07.31 .1992 & 90 & 12.30.1999 & 90 & $+106.06 \%$ & $+1.1917 \%$ & $3.6847 \%$ & $12.76 \%$ \\
\hline & bear & 90 & 12.30 .1999 & 127 & 01.31 .2003 & 38 & $-66.40 \%$ & $-1.6179 \%$ & $4.9219 \%$ & $17.05 \%$ \\
\hline & bull & 127 & 01.31 .2003 & 184 & 10.31 .2007 & 58 & $+63.35 \%$ & $+0.9208 \%$ & $2.6945 \%$ & $9.33 \%$ \\
\hline & bear & 184 & 10.31 .2007 & 200 & 02.27 .2009 & 17 & $-56.24 \%$ & $-3.0811 \%$ & $5.6654 \%$ & $19.63 \%$ \\
\hline & bull & 200 & 02.27 .2009 & 291 & 09.30 .2016 & 92 & $+12.90 \%$ & $+0.5526 \%$ & $3.7230 \%$ & $12.90 \%$ \\
\hline \multirow[t]{5}{*}{$\mathrm{SMI}_{m d}$} & bull & 1 & 07.31 .1992 & 73 & 07.31 .1998 & 73 & $+152.22 \%$ & $+2.1142 \%$ & $4.4424 \%$ & $15.39 \%$ \\
\hline & bear & 73 & 07.31 .1998 & 129 & 05.31 .2003 & 57 & $-70.15 \%$ & $-1.1528 \%$ & $5.8858 \%$ & $20.39 \%$ \\
\hline & bull & 129 & 05.31 .2003 & 179 & 05.31 .2007 & 51 & $+83.86 \%$ & $+1.6146 \%$ & $2.8644 \%$ & $9.92 \%$ \\
\hline & bear & 179 & 05.31 .2007 & 200 & 02.27 .2009 & 22 & $-70.05 \%$ & $-3.1734 \%$ & $4.1556 \%$ & $14.40 \%$ \\
\hline & bull & 200 & 02.27 .2009 & 291 & 09.30 .2016 & 92 & $+55.11 \%$ & $+0.4683 \%$ & $3.6076 \%$ & $12.50 \%$ \\
\hline
\end{tabular}

Note: The table contains detailed information about the identified bull and bear markets for six indices from five countries. The six indices are: DowJones and S\&P500 (USA each), Nikkei (Japan), DAX (Germany), FTSE (Great Britain) and SMI (Switzerland). The data are monthly closing prices and cover the period from July 31, 1992 until September 30, 2016. The table lists the identified market phases as well as the beginning and end of the respective market phase in form of the data point $i$ and the corresponding date. The date is indicated in the date format mm.dd.yyyy. For every market phase the number of observations (no. of. observ.) in level prices as well as the overall and mean return, the standard deviation and volatility in returns are added.

\section{References}

Bry, G. and C. Boschan (1971). Cyclical analysis of time series: Selected procedures and computer programs. New York: Columbia University Press. 
Candelon, B., J. Piplack, and S. Straetmans (2008). On measuring synchronization of bulls and bears: The case of east asia. Journal of Banking \& Finance 32(6), 1022-1035.

Chauvet, M. and S. Potter (2000). Coincident and leading indicators of the stock market. Journal of Empirical Finance 7(1), 87-111.

Coudert, V. and H. Raymond-Feingold (2011). Gold and financial assets: Are there any safe havens in bear markets. Economics Bulletin 31(2), 1613-1622.

Gonzalez, L., P. Hoang, J. G. Powell, and J. Shi (2006). Defining and dating bull and bear markets: Two centuries of evidence. Multinational Finance Journal 10(1/2), 81-116.

Hamilton, J. D. (1989). A new approach to the economic analysis of nonstationary time series and the business cycle. Econometrica 57(2), 357-384.

Hamilton, W. P. (1919). Stock market analysis. Wall Street Journal 9.

Hardouvelis, G. A. and P. Theodossiou (2002). The asymmetric relation between initial margin requirements and stock market volatility across bull and bear markets. The Review of Financial Studies 15(5), 1525-1559.

Kole, E. and D. van Dijk (2017). How to identify and forcast bull and bear markets? Journal of Applied Econometrics 32(1), 120-139.

Lunde, A. and A. Timmermann (2004). Duration dependence in stock prices: An analysis of bull and bear markets. Journal of Business \& Economic Statistics 22(3), 253-273.

Maheu, J. M. and T. H. McCurdy (2000). Identifying bull and bear markets in stock returns. Journal of Business \& Economic Statistics 18(1), 100-112.

Pagan, A. R. and K. A. Sossounov (2003). A simple framework for analysing bull and bear markets. Journal of Applied Econometrics 18(1), 23-46.

Wu, S.-J. and W.-M. Lee (2012). Predicting the u.s. bear stock market using the consumptionwealth ratio. Economics Bulletin 32(4), 3174-3181. 